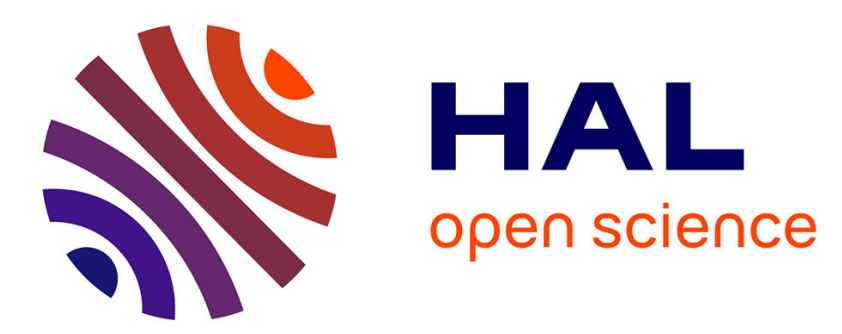

\title{
Stability control of a hybrid wheel-legged robot using potential field approach
}

Guillaume Besseron, Christophe Grand, Faïz Ben Amar, Philippe Bidaud

\section{To cite this version:}

Guillaume Besseron, Christophe Grand, Faïz Ben Amar, Philippe Bidaud. Stability control of a hybrid wheel-legged robot using potential field approach. Clawar'08: 11th International Conerence. on Climbing and Walking Robots, 2008, Coimbra, Portugal. pp.776-783. hal-03177939

\section{HAL Id: hal-03177939 \\ https://hal.science/hal-03177939}

Submitted on 29 Mar 2021

HAL is a multi-disciplinary open access archive for the deposit and dissemination of scientific research documents, whether they are published or not. The documents may come from teaching and research institutions in France or abroad, or from public or private research centers.
L'archive ouverte pluridisciplinaire HAL, est destinée au dépôt et à la diffusion de documents scientifiques de niveau recherche, publiés ou non, émanant des établissements d'enseignement et de recherche français ou étrangers, des laboratoires publics ou privés. 


\title{
STABILITY CONTROL OF A HYBRID WHEEL-LEGGED ROBOT USING POTENTIAL FIELD APPROACH
}

\author{
G. BESSERON, CH. GRAND, F. BEN AMAR and PH. BIDAUD \\ Institut des Systèmes Intelligents et de Robotique (ISIR) \\ Université Pierre \& Marie Curie - Paris VI, CNRS - FRE 2507 \\ Paris, France \\ E-mail: \{besseron, grand, amar, bidaud\}@isir.fr
}

\begin{abstract}
This paper concerns the control of an autonomous high mobility wheel-legged rover evolving on uneven terrains. A new control strategy, using active redundancies of the robot, leads to elaborate a posture control based on the potential field approach of the stability measurement. Then a decoupled posture and trajectory control algorithm based on the velocity model of the robot is proposed. Last, simulation results showing performance of the control algorithm are presented.
\end{abstract}

Keywords: wheel-legged robot, kinematic redundancy, stability margin, potential field, uneven terrain

\section{Introduction}

The main field of this research project deals with the mobility of autonomous robotic rovers navigating over an unknown natural environment. Many potential applications like planetary or extreme environment (volcanic, arctic or desert) exploration, agriculture, defense, demining, and others various missions in hazardous areas can be considered. Therefore autonomous mobile robots must be able to move on a wide variety of terrains while ensuring the integrity of the system (i.e. the stability holding to avoid tipover). The main difficulties in this kind of environment are due to the geometrical and physical soil properties (large slopes, roughness, rocks distribution, soil compaction, friction characteristics, etc).

High mobility hybrid systems - such as $\mathrm{Hylos}^{1}$, Workpartner ${ }^{2}$, or Athlete $^{3}$ robots - combine both advantages of wheeled and legged vehicles: the ability to ensure some higher velocity than legged systems for the first one, and to cross over terrains with high discontinuities (like rocks, steps, gaps, etc) for the second. 

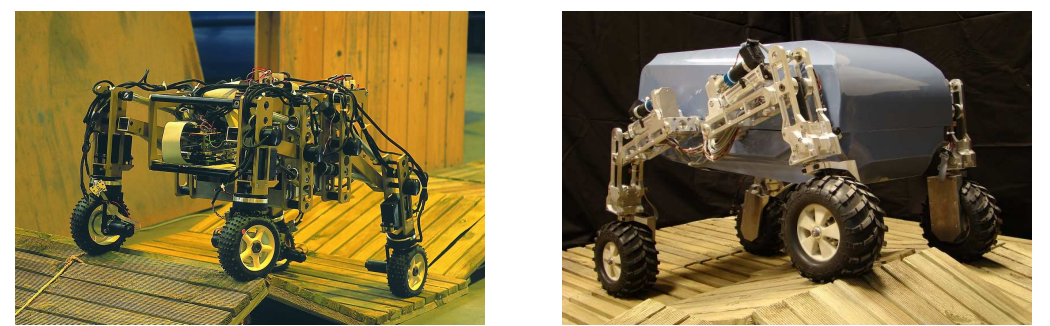

Fig. 1. Hylos I (left) and Hylos II (right)

In the paper, we propose to analyze the problem of stability control for the Hylos robots which were developed in our lab (see Fig. 1). These rovers are high mobility redundantly actuated hybrid systems. They are lightweight (around twenty-five kilogrammes) robots with sixteen actively actuated degrees-of-freedom. Because of active internal mobilities, the control of their posture should be considered. The problem of posture control for this kind of robotics system is quite challenging as the system exhibits complex dynamic interactions with the environment when evolving on roughly irregular terrain.

Control approaches of such redundantly actuated systems have been proposed in previous works ${ }^{4,5,1}$. In this study, the proposed posture control consists in modifying the robot posture in order to ensure its stability without specifying strictly a postural state. The posture correction is so made only when the stability of wheel-legged vehicle is jeopardized. The proposed controller is based on the technique of "potential fields" for which artificial potential reflecting the rover tipover stability margin is used.

In section 2, after the introduction of the used stability margin, the potential field based on stability measurement is proposed. Section 3 presents the formulation used to develop the kinematic model of a hybrid wheellegged robot. Next, decoupled posture and trajectory control algorithm is described in section 4. Finally, results of simulation to validate this new stability control strategy are shown in the last section 5 .

\section{Stability Margin and Linked Potential Field}

\subsection{Stability Margin}

The control method presented in this paper considers the vehicle movement on an irregular terrain without discontinuities. Thus, the tipover stability margin is mainly constrained by the terrain geometry. To ensure the integrity of the vehicle crossing over an uneven terrain, its stability margin 
index must be estimated all the time. The "tipover stability margin" proposed by Papadopoulos and Rey ${ }^{6}$ is used.

This tipover stability margin takes into account both the distance of the projected center-of-gravity (c.o.g) to the support polygon and its vertical position relatively to the average plane defined by contact points $C_{i}$. Moreover, all the external forces working on the c.o.g of the vehicle, including gravity, are considered. The formalism can be described briefly as follows (see Fig. 2): the line joining two consecutive terrain-contact points $C_{i}$ defines a tipover axis $\mathbf{a}_{i}$. The unit vector $\mathbf{h}_{i}$ of the axis joining the vehicle c.o.g, $G$, to the center of each tipover axis is computed. Then, the angle $v_{i}$ between each $\mathbf{h}_{i}$ and the tipover contribution $\boldsymbol{f}_{i}^{*}$, computed from the total external force $\boldsymbol{\tau}_{\boldsymbol{t}}=\left\{\boldsymbol{f}_{\boldsymbol{t}}, \boldsymbol{m}_{\boldsymbol{t}}\right\}$ applied to the vehicle, gives the stability angle over the corresponding tipover axis. Considering only quasi-static evolution of the vehicle here, the total external force $\tau_{t}$ is reduced to its own weight. The stability angle $v_{i}$ is therefore defined as the angle between $\mathbf{h}_{i}$ and the gravity resultant $\boldsymbol{g}$. The overall vehicle stability margin $m_{s}$ is also defined as the minimum of all stability angles $v_{i}$ :

$$
m_{s}=\min \left(v_{i}\right) \quad \text { for } \quad i=\{1, \ldots, n\}
$$

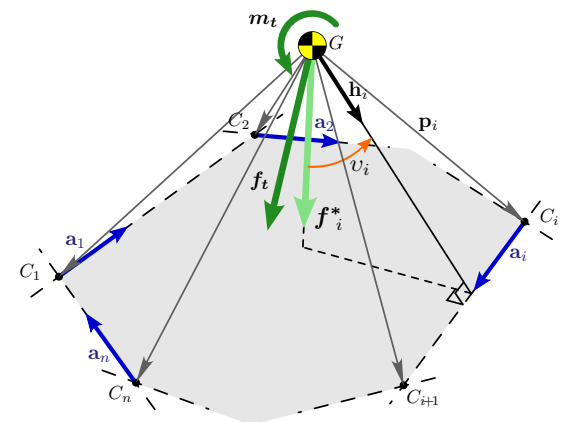

Fig. 2. Used stability margin by Papadopoulos and Rey

\subsection{Stability Potential Field}

In using the artificial potential field approach described by $\mathrm{Khatib}^{7}$, a stability potential function relative to the stability margin described in the previous section has been defined. This stability potential function $U_{s t a b}$ results from the sum of each $U_{s t a b_{i}}$, which specified to each stability angles $v_{i}$. The stability potential function $U_{s t a b}$ results from the sum of each $U_{s t a b_{i}}$, of which the specific form of repulsive potential function has been chosen in accordance with the potential field approach. 


$$
U_{s t a b}(\mathbf{q})=\sum_{i} U_{s t a b_{i}}(\mathbf{q})
$$

with

$$
U_{s t a b_{i}}(\mathbf{q})=\left\{\begin{array}{cc}
\frac{1}{2} k_{\text {stab }}\left(\frac{1}{v_{i}(\mathbf{q})}-\frac{1}{v^{*}}\right)^{2} & \text { if } v_{i} \leq v^{*} \\
0 & \text { if } v_{i}>v^{*}
\end{array}\right.
$$

where $v_{i}$ is the stability angle or tipover angle relative to $i^{\text {th }}$ tipover axis, $v^{*}$ is the stability angle limit. Thus, the threshold of stability measurement from action must be defined in order to maintain an acceptable stability. $k_{s t a b}$ is a constant gain.

\section{Kinematic Model}

In this paper, the same formalism as the one defined on previous works ${ }^{1,8}$ is used and adapted to the specific kinematics of the Hylos II robot.

The differential kinematic model is obtained by means of the velocity composition principle expressed in the contact frame $\mathcal{R}_{C_{i}}$ :

$$
\mathbf{v}_{\mathbf{s}}=-\mathbf{v}_{\mathbf{c}}+\mathbf{v}_{\mathbf{p}_{i}}+\mathbf{v}_{\mathbf{x}}
$$

where

- $\mathbf{v}_{\mathbf{s}}$ is the sliding velocity of the contact point $C_{i}$,

- $\mathbf{v}_{\mathbf{x}}$ is the velocity of $C_{i}$ due to platform motion with respect to ground,

- $\mathbf{v}_{\mathbf{p}_{i}}$ is the velocity of $C_{i}$ due to leg's motion with respect the platform,

- $\mathbf{v}_{\mathbf{c}}=r \omega_{i} \boldsymbol{t}_{i}$ is the wheel circumferential velocity with respect to the leg.

On the assumption of pure rolling (slip velocity is null), we then obtain from equation (4) by projection on the contact frame:

$$
\mathbf{R}_{i}^{\mathrm{t}} \mathbf{L}_{i} \dot{\mathbf{x}}+\mathbf{R}_{i}^{\mathrm{t}} \mathbf{J}_{\mathbf{p}_{i}} \dot{\boldsymbol{\theta}}_{i}-r \omega_{i} \boldsymbol{t}_{i}=\mathbf{0}
$$

where $\mathbf{R}_{i}$ is matrix rotation of contact frame with respect to platform frame and $\omega_{i}$ is the $i^{\text {th }}$ wheel rate.

Finally, we obtain, in matrix-form, the velocity equation for the whole system composed of four wheel-leg chains:

$$
\mathbf{L} \dot{\mathbf{x}}+\mathbf{J} \dot{\mathbf{q}}=\mathbf{0}
$$

where $\mathbf{L}$ is the locomotion matrix which gives the wheel contribution to the plateform movement, $\mathbf{J}$ corresponds to the Jacobian matrix of wheel-legged kinematic chain, and where $\mathbf{x}$ and $\mathbf{q}$ are respectively vectors of the platform parameters and the articular-joint parameters of wheel-legged chains. 


\section{Decoupled Control}

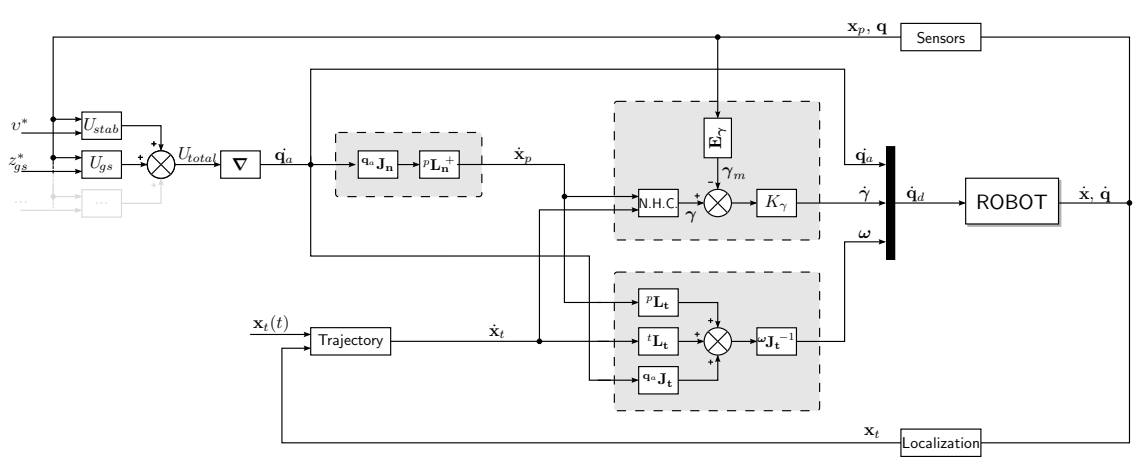

Fig. 3. Control scheme

The motion control of the studied redundant systems is based on the resolution of the inverse velocity model. Several classical approaches of redundancies control and issued from manipulators control have been considered ${ }^{9-12}$. In order to solve inverse model of equation (6), the task in the operational space is defined following two modalities: one relative to the robot posture and the other for the trajectory control.

Thus the vector of platform velocities $\dot{\mathbf{x}}=(\dot{x}, \dot{y}, \dot{z}, \dot{\varphi}, \dot{\psi}, \dot{\theta})^{t}-$ input of inverse model - is split into two sub-vectors $\dot{\mathbf{x}}_{t}$ and $\dot{\mathbf{x}}_{p}$ which put together respectively trajectory and posture terms:

$$
\dot{\mathbf{x}}_{t}=(\dot{x}, \dot{y}, \dot{\theta})^{t}=\mathbf{S}_{t} \dot{\mathbf{x}} \quad \text { and } \quad \dot{\mathbf{x}}_{p}=(\dot{z}, \dot{\varphi}, \dot{\psi})^{t}=\mathbf{S}_{p} \dot{\mathbf{x}}
$$

where $\mathbf{S}_{t}$ and $\mathbf{S}_{p}$ are appropriate sorting matrices.

Next the inverse kinematic model solution results from three consecutive steps. Each one corresponds to the resolution of system (6) projected onto one of the contact frame axis $\left(\boldsymbol{n}_{i}\right.$, then $\boldsymbol{t}_{i}$ and $\left.\boldsymbol{l}_{i}\right)$. These steps lead to determine successively the joint rates $\dot{\mathbf{q}}_{a}$ of the robot (i.e. $q_{1_{i}}$ and $q_{2_{i}}$ ), then the wheel rates $\boldsymbol{\omega}$, and finally the steering rates $\dot{\boldsymbol{\gamma}}$. The matrices $\mathbf{S}_{\mathbf{q}_{a}}, \mathbf{S}_{\boldsymbol{\gamma}}$ et $\mathbf{S}_{\boldsymbol{\omega}}$ are defined to sort respectively the joint parameters relative to the posture, the direction change and the wheel rotation:

$$
\dot{\mathbf{q}}_{a}=\mathbf{S}_{\mathbf{q}_{a}} \dot{\mathbf{q}} \quad \dot{\gamma}=\mathbf{S}_{\gamma} \dot{\mathbf{q}} \quad \boldsymbol{\omega}=\mathbf{S}_{\boldsymbol{\omega}} \dot{\mathbf{q}}
$$

At each step of this resolution, analyses are made, leading to simplify and to neglect some terms issued from sorting. Thus, the projection of equation (6) on $\mathbf{n}$, the set of vectors $\boldsymbol{n}_{i}$, gives the following equation:

$$
\mathbf{P}_{\mathbf{n}} \mathbf{L} \dot{\mathbf{x}}+\mathbf{P}_{\mathbf{n}} \mathbf{J} \dot{\mathbf{q}}=\mathbf{0}
$$


where $\mathbf{P}_{\mathbf{n}}$ is the projection matrix associated to $\mathbf{n}$. Appropriate simplifications give relation (10) : the terms relative to the trajectory are inconsiderable for small pitch and roll angles, those referring to wheel rates have no effect on the robot posture, and the weak influence of direction axis rates on instantaneous velocities field of the Hylos robot could be neglected.

$$
{ }^{p} \mathbf{L}_{\mathbf{n}} \dot{\mathbf{x}}_{p}+{ }^{\mathbf{q} a} \mathbf{J}_{\mathbf{n}} \dot{\mathbf{q}}_{a}=\mathbf{0}
$$

with ${ }^{p} \mathbf{L}_{\mathbf{n}}=\left(\mathbf{P}_{\mathbf{n}} \mathbf{L} \mathbf{S}_{p}{ }^{t}\right)$ and ${ }^{\mathbf{q}_{a}} \mathbf{J}_{\mathbf{n}}=\left(\mathbf{P}_{\mathbf{n}} \mathbf{J S}_{\mathbf{q}_{a}{ }^{t}}\right)$. This relation allows to determine the posture kinematic vector $\dot{\mathbf{x}}_{p}$ from the following equation:

$$
\begin{aligned}
\dot{\mathbf{x}}_{p} & =-{ }^{p} \mathbf{L}_{\mathbf{n}}+\mathbf{q}_{a} \mathbf{J}_{\mathbf{n}} \dot{\mathbf{q}}_{a} \\
& =-{ }^{p} \mathbf{L}_{\mathbf{n}}+\mathbf{q}_{a} \mathbf{J}_{\mathbf{n}} \nabla U
\end{aligned}
$$

where ${ }^{p} \mathbf{L}_{\mathbf{n}}{ }^{+}$represents the pseudo-inverse matrix of ${ }^{p} \mathbf{L}_{\mathbf{n}}$. The input of this reducted inverse kinematic model is $\dot{\mathbf{q}}_{a}$, which is substituted by the potential field gradient $\nabla U$, established previously in order to act on the robot posture (see Sec. 2).

Then the projection of the equation (6) on the set of $\boldsymbol{t}_{i}$ leads to equation (12), which, once simplifications made (Eq. 13), leads to the expression of $\boldsymbol{\omega}$ (Eq. (14)):

$$
\mathbf{P}_{\mathbf{t}} \mathbf{L} \dot{\mathbf{x}}+\mathbf{P}_{\mathbf{t}} \mathbf{J} \dot{\mathbf{q}}=\mathbf{0}
$$

For the same previous reason, the terms of direction axis rates are also ignored.

$$
\mathbf{P}_{\mathbf{t}} \mathbf{L} \dot{\mathbf{x}}+{ }^{\mathbf{q}_{a}} \mathbf{J}_{\mathbf{t}} \dot{\mathbf{q}}_{a}+{ }^{\boldsymbol{\omega}} \mathbf{J}_{\mathbf{t}} \boldsymbol{\omega}=\mathbf{0}
$$

with ${ }^{\mathbf{q}_{a}} \mathbf{J}_{\mathbf{t}}=\left(\mathbf{P}_{\mathbf{t}} \mathbf{J S}_{\mathbf{q}_{a}}{ }^{t}\right)$ and ${ }^{\boldsymbol{\omega}} \mathbf{J}_{\mathbf{t}}=\left(\mathbf{P}_{\mathbf{t}} \mathbf{J} \mathbf{S}_{\boldsymbol{\omega}}{ }^{t}\right)$. Then the posture control $\dot{\mathbf{x}}_{p}$, issued from the previous step of algorithm resolution (Eq. (11)), and the desired trajectory $\dot{\mathbf{x}}_{t}$ are inputed in the equation (14). As previously, $\dot{\mathbf{q}}_{a}$ has been substituted by the potential field gradient $\nabla U$.

$$
\boldsymbol{\omega}=-\left({ }^{\boldsymbol{\omega}} \mathbf{J}_{\mathbf{t}}\right)^{-1}\left({ }^{t} \mathbf{L}_{\mathbf{t}} \dot{\mathbf{x}}_{t}+{ }^{p} \mathbf{L}_{\mathbf{t}} \dot{\mathbf{x}}_{p}+{ }^{\mathbf{q}_{a}} \mathbf{J}_{\mathbf{t}} \nabla U\right)
$$

The last step leads to determine the steering rate $\dot{\gamma}_{i}$. In resolving the non-holonomic constraints (N.H.C.), the desired steering angles $\gamma_{i}$ are determined. A simple proportional controller based on the positioning error is used to compute the rate $\dot{\gamma}$.

$$
\dot{\gamma}=\left\{\dot{\gamma}_{i}\right\} \quad \text { with } \quad \dot{\gamma}_{i}=K_{\gamma}\left(\gamma_{i}-\gamma_{i_{m}}\right)
$$

where $\gamma_{i_{m}}$ is the measure of the steering angle, and $K_{\gamma}$ is a gain control.

The whole posture control algorithm is summed up through the control scheme depicted in figure 3 . 


\section{Results}

In the first time, the proposed posture control algorithm has been evaluated in simulation in order to validate its running principle. As shown in figure 4, this one has consisted in modeling kinematics behaviour of the Hylos robot in a sinuous elevation ground with a loop trajectory (going from point "A" to point "B"). The results of this simulation are presented through figures 5 and 6 , which depict respectively the evolution of the stability margin and the global potential generated for the posture correction.

As planned, a posture correction of the robot is made when the stability margin dropes under the stability margin limit. Every time that this case appears, the potential function relative to the stability measurement becomes non-null (see Fig. 6). In line with the equation (11), this generated potential acts directly on the robot posture in order to preserve its stability. Except a slight excess when the first correction is needed just after 2.7 seconds of simulation, until the posture correction has been efficient, the stability margin does not jut out above the stability margin limit (see Fig. 5). Thus the robot stability is preserved without imposing a specific posture.

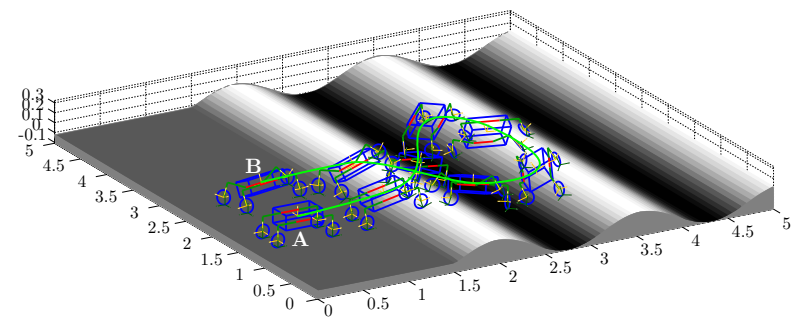

Fig. 4. Robot simulation on a sinuous elevation terrain with a "loop" trajectory

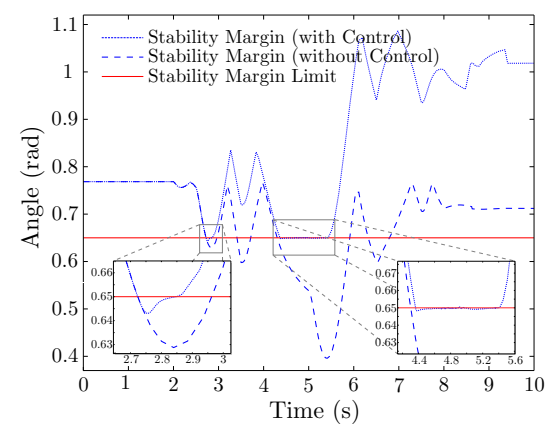

Fig. 5. Stability margin

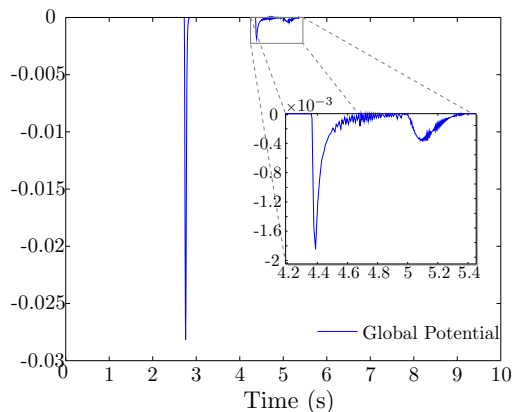

Fig. 6. Global Potential 


\section{Conclusion and Future Works}

In this paper, a new stability control strategy for a wheel-legged robot has been proposed. This one is come from the idea to use active redundancies of the studied rover in order to ensure its stability without imposing a particular posture, as made in previous works ${ }^{1}$. This strategy needed to set up a decoupled control of posture and trajectory. An original velocity based control algorithm has been presented. This approach allows to carry out the desired behaviour of the robot. The algorithm has been validated through simulations, showing the capabilities of a such redundantly actuated robot to ensure both its stability margin during the whole motion on uneven terrain and a specified trajectory.

As future works, the stability measurement is solved with considering the dynamics of the system. Experiments with the Hylos robot are also in progress. Shortly the practical feasibility of this control approach will be evaluated and validated through these experiments.

\section{References}

1. C. Grand, F. BenAmar, F. Plumet and P. Bidaud, Int. Journal of Robotics Research 23, 1041 (2004).

2. A. Halme, I. Leppänen, S. Salmi and S. Ylönen, Hybrid locomotion of a wheel-legged machine, in Int. Conf. on Climbing and Walking Robots, 2000.

3. B. H. Wilcox, T. Litwin, J. Biesiadecki, J. Matthews, M. Heverly, J. Morrison, J. Townsend, N. Ahmed, A. Sirota and B. Cooper, Journal of Field Robotics 24, 421(April 2007).

4. S. Sreenivasan and B. Wilcox, Journal of Robotics Systems 11, 487 (1994).

5. K. Iagnemma, A. Rzepniewski, S. Dubowsky and P. Schenker, Autonomous Robots 14, 5 (2003).

6. E. Papadopoulos and D. Rey, A new mesure of tipover stability for mobile manipulators, in Int. Conf. on Robotics and Automation, 1996.

7. O. Khatib, Int. Journal of Robotics Research 5, 90 (1986).

8. C. Grand, F. B. Amar and F. Plumet, Motion kinematics analysis of wheeledlegged rover over 3d surface with posture adaptation, in 12th IFToMM World Congress in Mechanism and Machine Science, (Besançon, France, 2007).

9. J. Baillieul, Kinematic programming alternatives for redundant manipulators, in Int. Conference on Robotics and Automation, (St Louis, US, 1985).

10. D. N. Nenchev, Int. Journal of Robotics Research 11, 584 (1992).

11. C. A. Klein, C. Chu-Jenq and S. Ahmed, Transactions on Robotics and Automation 11, 50 (1995).

12. J. Lenarčič, Effective secondary task execution of redundant manipulators, in Robotica, ed. C. U. PressJuly 1998. 